\title{
ON THE WAVE OPERATORS FOR THE CRITICAL NONLINEAR SCHRÖDINGER EQUATION
}

\author{
RÉmi Carles and Tohru Ozawa
}

\begin{abstract}
We prove that for the $L^{2}$-critical nonlinear Schrödinger equations, the wave operators and their inverse are related explicitly in terms of the Fourier transform. We discuss some consequences of this property. In the one-dimensional case, we show a precise similarity between the $L^{2}$-critical nonlinear Schrödinger equation and a nonlinear Schrödinger equation of derivative type.
\end{abstract}

\section{Introduction}

We consider the defocusing, $L^{2}$-critical, nonlinear Schrödinger equation

$$
i \partial_{t} u+\frac{1}{2} \Delta u=|u|^{4 / n} u, \quad(t, x) \in \mathbb{R} \times \mathbb{R}^{n} .
$$

We consider two types of initial data:

$$
\begin{aligned}
& \text { Asymptotic state: }\left.U_{0}(-t) u(t)\right|_{t= \pm \infty}=u_{ \pm}, \quad \text { where } U_{0}(t)=e^{i \frac{t}{2} \Delta} . \\
& \text { Cauchy data at } t=0: u_{\mid t=0}=u_{0} .
\end{aligned}
$$

It is well known that for data $u_{ \pm}, u_{0} \in \Sigma=H^{1} \cap \mathcal{F}\left(H^{1}\right)$, where

$$
\mathcal{F} f(\xi)=\widehat{f}(\xi)=\frac{1}{(2 \pi)^{n / 2}} \int_{\mathbb{R}^{n}} f(x) e^{-i x \cdot \xi} d x,
$$

1.1)-1.2 has a unique, global, solution $u \in C(\mathbb{R} ; \Sigma)$ ([11], see also [5]). Its initial value $u_{\mid t=0}$ is the image of the asymptotic state under the action of the wave operator:

$$
u_{\mid t=0}=W_{ \pm} u_{ \pm} .
$$

Similarly, 1.1 -1.3 possesses asymptotic states:

$$
\exists u_{ \pm} \in \Sigma, \quad\left\|U_{0}(-t) u(t)-u_{ \pm}\right\|_{\Sigma} \underset{t \rightarrow \pm \infty}{\longrightarrow} 0: \quad u_{ \pm}=W_{ \pm}^{-1} u_{0} .
$$

Global well-posedness properties show that the wave operators are homeomorphisms on $\Sigma$. Besides this point, very few properties of these operators are known. The main result of this paper (proved in $\$ 2$ see also Appendix $\mathrm{A}$ for an alternate proof) shows that the wave operators and their inverses are related in terms of the Fourier transform:

Theorem 1.1. Let $n \geqslant 1$. The following identity holds on $\Sigma$ :

$$
\mathcal{F} \circ W_{ \pm}^{-1}=W_{\mp} \circ \mathcal{F} \text {. }
$$

Received by the editors February 27, 2007.

2000 Mathematics Subject Classification. 35B33; 35B40; 35P25; 35Q55. 
In particular, if $\mathcal{C}$ denotes the conjugation $f \mapsto \bar{f}$, then we have:

$$
W_{ \pm}^{-1}=(\mathcal{C F})^{-1} W_{ \pm}(\mathcal{C F}) .
$$

Using continuity properties of the flow map associated to $(1.1)$, we infer the following result in 3 .

Corollary 1.2. The result of Theorem 1.1 still holds when $\Sigma$ is replaced

- Either by $\mathcal{F}\left(H^{1}\right)$,

- Or by a neighborhood of the origin in $L^{2}\left(\mathbb{R}^{n}\right)$, for 11.1 as well as for its focusing counterpart, $i \partial_{t} u+\frac{1}{2} \Delta u=-|u|^{4 / n} u$,

- Or by $L_{r}^{2}\left(\mathbb{R}^{n}\right)$ for $n \geqslant 3$, the set of radial, square integrable functions.

Remark 1.3. The usual conjecture on (1.1) implies that the result of Theorem 1.1 is expected to remain valid when $\Sigma$ is replaced by $L^{2}\left(\mathbb{R}^{n}\right)$ (but not for the focusing counterpart of (1.1), for which finite time blow-up may occur in $H^{1}$ ).

Remark 1.4. So far, the existence of wave operators on $\mathcal{F}\left(H^{1}\right)$ is not known. Similarly, asymptotic completeness in $H^{1}$ remains an open problem. Theorem 1.1 shows that the fact that these two problems are simultaneously open is not merely a technical point: they are exactly related by (1.4). This aspect is also reminiscent of the main result in [1].

Using the asymptotic expansion of the wave operators near the origin, we prove in $\$ 4$ (with an extension in Appendix B):

Corollary 1.5. Let $n \geqslant 1$. For every $\phi \in L^{2}\left(\mathbb{R}^{n}\right)$, we have:

$$
\int_{0}^{ \pm \infty} e^{i t \frac{|x|^{2}}{2}} \mathcal{F}\left(\left|U_{0}(t) \phi\right|^{4 / n} U_{0}(t) \phi\right) d t=\int_{0}^{ \pm \infty} U_{0}(t)\left(\left|U_{0}(-t) \widehat{\phi}\right|^{4 / n} U_{0}(-t) \widehat{\phi}\right) d t .
$$

Finally, in space dimension $n=1$, we relate the wave operators for 1.1 with the wave operators for the nonlinear Schrödinger equation of derivative type

$$
i \partial_{t} \psi+\frac{1}{2} \partial_{x}^{2} \psi=i \lambda \partial_{x}\left(|\psi|^{2}\right) \psi, \quad \lambda \in \mathbb{R} .
$$

This equation appears as a model to study the nonlinear self-modulation for the Benjamin-Ono equation [16]. For a more general nonlinear Schrödinger equation of derivative type (see e.g. 13, 19] for the Cauchy problem related to similar equations),

$$
i \partial_{t} \psi+\frac{1}{2} \partial_{x}^{2} \psi=i \lambda|\psi|^{2} \partial_{x} \psi+i \mu \psi^{2} \partial_{x} \bar{\psi},
$$

it is proved in 14 that a short range scattering theory is available for $\lambda, \mu \in \mathbb{R}$ if and only if $\lambda=\mu$ : we recover 1.6 . This is apparently the only cubic, gauge invariant nonlinearity in space dimension one, for which a short range scattering theory is available. More precisely, for $1.6-\sqrt{1.2}$, the wave operators $\Omega_{ \pm}: u_{ \pm} \mapsto u(0)$ are well defined from $X_{\varepsilon}$ to $H^{2}(\mathbb{R})$, where

$$
X_{\varepsilon}=\left\{\phi \in H^{4} \cap \mathcal{F}\left(H^{4}\right) \quad ; \quad\left\|\left(1+\xi^{2}\right) \hat{\phi}\right\|_{L^{\infty}}<\varepsilon\right\},
$$

and $\varepsilon>0$ is sufficiently small. The following result shows that the nonlinearity in (1.6) should be thought of as the quintic case (1.1). This result goes in the same spirit as the approach followed in [15]. 
Theorem 1.6. Let $\lambda \in \mathbb{R}$. Consider the quintic, focusing or defocusing, equation

$$
i \partial_{t} u+\frac{1}{2} \partial_{x}^{2} u=\frac{\lambda^{2}}{2}|u|^{4} u, \quad(t, x) \in \mathbb{R} \times \mathbb{R},
$$

with associated wave operators $W_{ \pm}(\lambda)$ for small $L^{2}$ data. For $\phi \in L^{2}(\mathbb{R})$, define

$$
\left(N_{ \pm}^{\lambda} \phi\right)(x)=\phi(x) \exp \left( \pm i \lambda \int_{-\infty}^{x}|\phi(y)|^{2} d y\right) .
$$

- If $\psi$ solves 1.6 , then $N_{-}^{\lambda}(\psi)$ solves (1.7).

- If u solves (1.7), then $N_{+}^{\lambda}(u)$ solves (1.6).

- The following identity holds when all terms are well-defined:

$$
\begin{aligned}
& \mathcal{F} \circ \Omega_{ \pm}^{-1}=N_{-}^{\lambda} \circ \mathcal{F} \circ W_{ \pm}^{-1} \circ N_{+}^{\lambda}=\left(N_{+}^{\lambda}\right)^{-1} \circ \mathcal{F} \circ W_{ \pm}^{-1} \circ N_{+}^{\lambda} \\
& \Omega_{ \pm} \circ \mathcal{F}^{-1}=\left(N_{+}^{\lambda}\right)^{-1} \circ W_{ \pm} \circ \mathcal{F}^{-1} \circ N_{+}^{\lambda} .
\end{aligned}
$$

This result is checked by elementary computations, so we leave out its proof.

\section{Proof of Theorem 1.1}

2.1. Formal proof. Introduce the transform $\Psi$ acting on function of $(t, x)$ as:

$$
(\Psi u)(t, x)=\frac{1}{(i t)^{n / 2}} e^{i \frac{|x|^{2}}{2 t}} u\left(\frac{-1}{t}, \frac{x}{t}\right), \quad \text { for } t \neq 0 .
$$

Because the critical nonlinear Schrödinger equation (1.1) is also conformally invariant, $\Psi$ maps a solution to 1.1 to another solution to (1.1). Let $v=\Psi u$. It satisfies

$$
i \partial_{t} v+\frac{1}{2} \Delta v=|v|^{4 / n} v, \quad(t, x) \in \mathbb{R} \backslash\{0\} \times \mathbb{R}^{n} .
$$

The Cauchy datum for $u$ at time $t=0$ corresponds to scattering data for $v$ :

$$
U_{0}(-t) v(t) \underset{t \rightarrow \pm \infty}{\longrightarrow} \mathcal{F}^{-1} u_{0} \text {. }
$$

Therefore, we have

$$
v\left(0^{ \pm}\right)=W_{ \pm}\left(\mathcal{F}^{-1} u_{0}\right)
$$

Define

$$
\widetilde{u}(t, x)=U_{0}(-t) u(t, x) \quad ; \quad \widetilde{v}(t, x)=U_{0}(-t) v(t, x) .
$$

Denoting by $\mathcal{M}_{t}$ the multiplication by $e^{i|x|^{2} /(2 t)}$, and by $D_{t}$ the dilation operator

$$
D_{t} \phi(x)=\frac{1}{(i t)^{n / 2}} \phi\left(\frac{x}{t}\right)
$$

we recall the classical decomposition, for $t \neq 0$ :

$$
U_{0}(t)=\mathcal{M}_{t} D_{t} \mathcal{F} \mathcal{M}_{t} .
$$

It shows that the functions $\widetilde{u}$ and $\widetilde{v}$ are related by the relation

$$
\widetilde{u}(t)=\mathcal{M}_{t}^{-1} \mathcal{F}^{-1} v\left(\frac{-1}{t}\right)=\mathcal{F}^{-1} \widetilde{v}\left(\frac{-1}{t}\right) .
$$

This yields

$$
v\left(0^{ \pm}\right)=\widetilde{v}\left(0^{ \pm}\right)=\mathcal{F}\left(\lim _{t \rightarrow \mp \infty} \widetilde{u}(t)\right)=\mathcal{F}\left(W_{\mp}^{-1} u_{0}\right) .
$$


Then (2.2) and (2.4) yield (1.4), at least formally.

Remark 2.1. As pointed out by the referee, we could replace $v=\Psi u$ by the pseudoconformal inverse $u_{c}$ defined by

$$
u_{c}(t, x)=\frac{1}{(i t)^{n / 2}} e^{i \frac{|x|^{2}}{2 t}} \bar{u}\left(\frac{1}{t}, \frac{x}{t}\right), \quad \text { for } t \neq 0,
$$

that is,

$$
u_{c}(t)=\mathcal{M}_{t} D_{t} \bar{u}\left(\frac{1}{t}\right)
$$

Then the intuitive proof, as well as the rigorous one, follow the same lines.

2.2. Rigorous proof. The rigorous proof of Theorem 1.1 relies on a series of lemmas, which are stated, and proved, in a slightly different fashion in [18].

Lemma 2.2. For $n \geqslant 1$ and $\phi \in L^{2}\left(\mathbb{R}^{n}\right)$, we have:

$$
\lim _{t \rightarrow \pm \infty}\left\|U_{0}(t) \mathcal{F}^{-1} \phi(\cdot)-(\Psi \phi)(t, \cdot)\right\|_{L^{2}}=0 \text {. }
$$

Proof. With 2.3 in mind, we note that $\Psi \phi=\mathcal{M} D \phi$. Plancherel formula yields:

$$
\left\|U_{0}(t) \mathcal{F}^{-1} \phi(\cdot)-(\Psi \phi)(t, \cdot)\right\|_{L^{2}}=\left\|\left(M_{t}-1\right) \mathcal{F}^{-1} \phi(\cdot)\right\|_{L^{2}} .
$$

Since $\left|M_{t}(x)-1\right| \lesssim|x| / \sqrt{t}$, the lemma follows for $\phi \in H^{1}\left(\mathbb{R}^{n}\right)$. By density, we infer the result for $\phi \in L^{2}\left(\mathbb{R}^{n}\right)$.

Lemma 2.3. Let $v=\Psi u$. Suppose that there exist $\psi_{ \pm} \in L^{2}\left(\mathbb{R}^{n}\right)$ such that

$$
\left\|v(t)-\psi_{ \pm}\right\|_{L^{2}} \underset{t \rightarrow 0}{\longrightarrow} 0 .
$$

Then $u$ has asymptotic states in $L^{2}$ :

$$
\left\|U_{0}(-t) u(t)-\mathcal{F}^{-1} R \psi_{\mp}\right\|_{L^{2}} \underset{t \rightarrow \pm \infty}{\longrightarrow} 0,
$$

where $R$ stands for the symmetry with respect to the origin, $(R \phi)(x)=\phi(-x)$.

Proof. We note that $\Psi$ is almost an involution: $\Psi^{2}=R$. Therefore, $u=\Psi R v$ :

$$
\begin{aligned}
U_{0}(-t) u(t)-\mathcal{F}^{-1} R \psi_{\mp} & =U_{0}(-t) \Psi R v\left(\frac{-1}{t}\right)-\mathcal{F}^{-1} R \psi_{\mp} \\
& =U_{0}(-t) \Psi R\left(v\left(\frac{-1}{t}\right)-\psi_{\mp}\right)+\left(U_{0}(-t) \Psi-\mathcal{F}^{-1}\right) R \psi_{\mp} .
\end{aligned}
$$

Taking the $L^{2}$ norm, we infer:

$$
\left\|U_{0}(-t) u(t)-\mathcal{F}^{-1} R \psi_{\mp}\right\|_{L^{2}} \leqslant\left\|v\left(\frac{-1}{t}\right)-\psi_{\mp}\right\|_{L^{2}}+\left\|\Psi R \psi_{\mp}-U_{0}(t) \mathcal{F}^{-1} R \psi_{\mp}\right\|_{L^{2}} .
$$

The first term of the right-hand side goes to zero as $t \rightarrow \pm \infty$ by assumption. The second term goes to zero by Lemma 2.2

Lemma 2.4. Let $v=\Psi u$. Suppose that $u \in C\left([-T, T] ; L^{2}\right)$ for some $T>0$, and $u_{\mid t=0}=u_{0} \in L^{2}\left(\mathbb{R}^{n}\right)$. Then

$$
\left\|U_{0}(-t) v(t)-\mathcal{F}^{-1} u_{0}\right\|_{L^{2}} \underset{t \rightarrow \pm \infty}{\longrightarrow} 0 .
$$


Proof. Since $U_{0}(-t)=U_{0}(t)^{-1}$, we have

$$
U_{0}(-t) v(t)=\mathcal{M}_{t}^{-1} \mathcal{F}^{-1} D_{t}^{-1} \mathcal{M}_{t}^{-1} v(t)=\mathcal{M}_{t}^{-1} \mathcal{F}^{-1} u\left(\frac{-1}{t}\right) .
$$

Therefore,

$$
\left\|U_{0}(-t) v(t)-\mathcal{F}^{-1} u_{0}\right\|_{L^{2}} \leqslant\left\|u\left(\frac{-1}{t}\right)-u_{0}\right\|_{L^{2}}+\left\|\left(\mathcal{M}_{-t}-1\right) \mathcal{F}^{-1} u_{0}\right\|_{L^{2}} .
$$

The first term of the right-hand side goes to zero as $t \rightarrow \pm \infty$ by assumption. So does the second, by the standard argument recalled in the proof of Lemma 2.2 .

Proof of Theorem 1.1. Let $u_{0} \in \Sigma$ : there exists a unique solution $u \in C(\mathbb{R} ; \Sigma)$ to (1.1)-1.3. Set $v=\Psi u$. Because of the conformal invariance for (1.1), $v$ solves the same equation as $u$, for $t \neq 0$ :

$$
i \partial_{t} v+\frac{1}{2} \Delta v=|v|^{4 / n} v, \quad(t, x) \in \mathbb{R} \backslash\{0\} \times \mathbb{R}^{n} .
$$

Lemma 2.4 shows that

$$
\left\|U_{0}(-t) v(t)-\mathcal{F}^{-1} u_{0}\right\|_{L^{2}} \underset{t \rightarrow \pm \infty}{\longrightarrow} 0 .
$$

Let $w_{ \pm}$denote the solutions to the scattering problems:

$$
i \partial_{t} w_{ \pm}+\frac{1}{2} \Delta w_{ \pm}=\left|w_{ \pm}\right|^{4 / n} w_{ \pm} \quad ;\left.\quad U_{0}(-t) w_{ \pm}(t)\right|_{t= \pm \infty}=\mathcal{F}^{-1} u_{0} .
$$

By uniqueness for $1.1-1.2$, we see that

$$
v(t, x)= \begin{cases}w_{-}(t, x) & \text { for } t<0 \\ w_{+}(t, x) & \text { for } t>0\end{cases}
$$

In particular,

$$
\left\|v(t)-w_{ \pm}(0)\right\|_{L^{2}} \underset{t \rightarrow \pm 0}{\longrightarrow} 0 .
$$

From Lemma $2.3 u$ has asymptotics states, given by:

$$
\left\|U_{0}(-t) u(t)-\mathcal{F}^{-1} R w_{\mp}(0)\right\|_{L^{2}} \underset{t \rightarrow \pm \infty}{\longrightarrow} 0,
$$

that is, $u_{ \pm}(\cdot)=\mathcal{F}^{-1} R w_{\mp}(0, \cdot)$. We infer:

$$
\mathcal{F} \circ W_{ \pm}^{-1} u_{0}=\mathcal{F} u_{ \pm}=R w_{\mp}(0)=R W_{\mp} \mathcal{F}^{-1} u_{0} .
$$

Since 1.1 is invariant by $R, R W_{\mp} \mathcal{F}^{-1} u_{0}=W_{\mp} R \mathcal{F}^{-1} u_{0}=W_{\mp} \mathcal{F} u_{0}$. This yields (1.4. The identity (1.5) follows from (1.4) and from the identity

$$
W_{ \pm}=\mathcal{C} \circ W_{\mp} \circ \mathcal{C},
$$

which was noticed in [7] (see also [5]). 


\section{Proof of Corollary 1.2}

The first case follows by density, since $W_{ \pm}$are defined and continuous on $H^{1}\left(\mathbb{R}^{n}\right)$ 12. (see also 9] for a simplified presentation), and since $W_{ \pm}^{-1}$ are defined and continuous on $\mathcal{F}\left(H^{1}\right)$ [10, 11, 18].

For the second case, existence of wave operators, their asymptotic completeness, and continuity properties, were proved by T. Cazenave and F. Weissler 6]. We note that Corollary 1.2 can be proved in this case like Theorem 1.1, provided that we work in a sufficiently small neighborhood of the origin in $L^{2}\left(\mathbb{R}^{n}\right)$.

The last case follows from the recent paper by T. Tao, M. Visan and X. Zhang [17. The proof of Corollary 1.2 then relies on asymptotic completeness (in the same space), along with continuous dependence upon the initial data. For $n \geqslant 3$, let $X=L_{r}^{2}\left(\mathbb{R}^{n}\right)$; $X$ is invariant under the action of the Fourier transform. For $\phi \in X$, let $\phi_{j}$ be a sequence in $\Sigma$, converging to $\phi$ in $X$. Define $u_{j}^{ \pm}$as the solutions to:

$$
i \partial_{t} u_{j}^{ \pm}+\frac{1}{2} \Delta u_{j}^{ \pm}=\left|u_{j}^{ \pm}\right|^{4 / n} u_{j}^{ \pm} \quad ;\left.\quad U_{0}(-t) u_{j}^{ \pm}(t)\right|_{t= \pm \infty}=\widehat{\phi}_{j} .
$$

There exists $u_{0 j}^{ \pm}=u_{j}^{ \pm}(0)=W_{ \pm} \widehat{\phi}_{j} \in \Sigma$. Since $u_{0 j}^{ \pm}=\mathcal{F} W_{\mp}^{-1} \phi_{j}$ from Theorem 1.1 the results in [6, 17] imply that there exists $u_{0}^{ \pm} \in X$ such that $\left\|u_{0 j}^{ \pm}-u_{0}^{ \pm}\right\|_{L^{2}} \rightarrow 0$ as $j \rightarrow \infty$. Let $u^{ \pm}$solve

$$
i \partial_{t} u^{ \pm}+\frac{1}{2} \Delta u^{ \pm}=\left|u^{ \pm}\right|^{4 / n} u^{ \pm} \quad ; \quad u_{\mid t=0}^{ \pm}=u_{0}^{ \pm} .
$$

We have

$$
\begin{aligned}
\left\|U_{0}(-t) u^{ \pm}(t)-\widehat{\phi}\right\|_{L^{2}} \leqslant & \left\|U_{0}(-t)\left(u^{ \pm}(t)-u_{j}^{ \pm}(t)\right)\right\|_{L^{2}}+\left\|U_{0}(-t) u_{j}^{ \pm}(t)-\widehat{\phi}_{j}\right\|_{L^{2}} \\
& +\left\|\phi_{j}-\phi\right\|_{L^{2}} .
\end{aligned}
$$

The global well-posedness for 1.1 in $X$ implies

$$
\limsup _{t \rightarrow \pm \infty}\left\|U_{0}(-t) u^{ \pm}(t)-\widehat{\phi}\right\|_{L^{2}} \leqslant F\left(\left\|u_{0}^{ \pm}-u_{0 j}^{ \pm}\right\|_{L^{2}}\right)+\left\|\phi_{j}-\phi\right\|_{L^{2}}
$$

where $F$ is a continuous function such that $F(0)=0$. Finally, by letting $j \rightarrow \infty$, we see that $u^{ \pm}$solves

$$
i \partial_{t} u^{ \pm}+\frac{1}{2} \Delta u^{ \pm}=\left|u^{ \pm}\right|^{4 / n} u^{ \pm} \quad ;\left.\quad U_{0}(-t) u^{ \pm}(t)\right|_{\mid t= \pm \infty}=\widehat{\phi} .
$$

Let $\mathcal{V}$ be a neighborhood of $\phi$ in $L^{2}$. From [6], we see by Strichartz estimates and a bootstrap argument that the problem $[1.1)-(1.2)$ is well-posed in $\left.\left.L^{\infty}(]-\infty,-T\right] ; \mathcal{V}\right)$ (we consider only the minus sign for simplicity) for some $T>0$ possibly depending on $\mathcal{V}$. By uniqueness, we infer

$$
\exists W_{ \pm} \widehat{\phi}=u_{0}^{ \pm} .
$$

Since under our assumptions, $W_{ \pm}^{-1}$ are homeomorphisms on $X$, we also have:

$$
u_{0}^{ \pm}=\lim _{j \rightarrow \infty} u_{0 j}^{ \pm}=\lim _{j \rightarrow \infty} \mathcal{F} W_{\mp}^{-1} \phi_{j}=\mathcal{F} W_{\mp}^{-1} \lim _{j \rightarrow \infty} \phi_{j}=\mathcal{F} W_{\mp}^{-1} \phi,
$$

hence Corollary 1.2 . 


\section{Proof of Corollary 1.5}

Corollary 1.5 is a consequence of Theorem 1.1 and of the asymptotic expansion of the wave operators near the origin in $L^{2}$ :

Proposition 4.1. Let $n \geqslant 1$ and $\phi \in L^{2}\left(\mathbb{R}^{n}\right)$. Then for $\varepsilon>0$ sufficiently small $W_{ \pm}\left(\varepsilon^{n / 4} \phi\right)$ and $W_{ \pm}^{-1}\left(\varepsilon^{n / 4} \phi\right)$ are well defined in $L^{2}\left(\mathbb{R}^{n}\right)$, and, as $\varepsilon \rightarrow 0$ :

$$
\begin{aligned}
& W_{ \pm}\left(\varepsilon^{\frac{n}{4}} \phi\right)=\varepsilon^{\frac{n}{4}} \phi+i \varepsilon^{1+\frac{n}{4}} \int_{0}^{ \pm \infty} U_{0}(-t)\left(\left|U_{0}(t) \phi\right|^{4 / n} U_{0}(t) \phi\right) d t+\mathcal{O}\left(\varepsilon^{2+\frac{n}{4}}\right) \\
& W_{ \pm}^{-1}\left(\varepsilon^{\frac{n}{4}} \phi\right)=\varepsilon^{\frac{n}{4}} \phi-i \varepsilon^{1+\frac{n}{4}} \int_{0}^{ \pm \infty} U_{0}(-t)\left(\left|U_{0}(t) \phi\right|^{4 / n} U_{0}(t) \phi\right) d t+\mathcal{O}\left(\varepsilon^{2+\frac{n}{4}}\right) .
\end{aligned}
$$

Proof. The proof follows from the same perturbative analysis as in [8] (see also [3] for the nonlinear Schrödinger equation). First, it follows from [6] that $W_{ \pm}\left(\varepsilon^{n / 4} \phi\right)$ and $W_{ \pm}^{-1}\left(\varepsilon^{n / 4} \phi\right)$ are well defined in $L^{2}\left(\mathbb{R}^{n}\right)$ for $\varepsilon>0$ sufficiently small.

We prove the asymptotic formula for the minus sign, since the proof of the formula for the plus sign is similar. Consider $u^{\varepsilon}$ solving:

$$
i \partial_{t} u^{\varepsilon}+\frac{1}{2} \Delta u^{\varepsilon}=\left|u^{\varepsilon}\right|^{4 / n} u^{\varepsilon} \quad ;\left.\quad U_{0}(-t) u^{\varepsilon}(t)\right|_{t=-\infty}=\varepsilon^{n / 4} \phi .
$$

Plugging an expansion of the form $u^{\varepsilon}=\varepsilon^{n / 4}\left(\varphi_{0}+\varepsilon \varphi_{1}+\varepsilon r^{\varepsilon}\right)$ into the above equation, and ordering in powers of $\varepsilon$, it is natural to impose the following conditions:

- Leading order: $\mathcal{O}\left(\varepsilon^{n / 4}\right)$.

$$
i \partial_{t} \varphi_{0}+\frac{1}{2} \Delta \varphi_{0}=0 \quad ;\left.\quad U_{0}(-t) \varphi_{0}(t)\right|_{t=-\infty}=\phi .
$$

- First corrector: $\mathcal{O}\left(\varepsilon^{1+n / 4}\right)$.

$$
i \partial_{t} \varphi_{1}+\frac{1}{2} \Delta \varphi_{1}=\left|\varphi_{0}\right|^{4 / n} \varphi_{0} \quad ;\left.\quad U_{0}(-t) \varphi_{1}(t)\right|_{t=-\infty}=0 .
$$

The first equation yields

$$
\varphi_{0}(t)=U_{0}(t) \phi .
$$

From the second equation, we have:

$$
\varphi_{1}(t)=-i \int_{-\infty}^{t} U_{0}(t-s)\left(\left|\varphi_{0}(s)\right|^{4 / n} \varphi_{0}(s)\right) d s .
$$

We also have:

$$
i \partial_{t} r^{\varepsilon}+\frac{1}{2} \Delta r^{\varepsilon}=G\left(\varphi_{0}+\varepsilon \varphi_{1}+\varepsilon r^{\varepsilon}\right)-G\left(\varphi_{0}\right) \quad ;\left.\quad U_{0}(-t) r^{\varepsilon}(t)\right|_{t=-\infty}=0,
$$


where $G(z)=|z|^{4 / n} z$. Let $\gamma=2+4 / n$, and denote $\left.\left.L_{t, x}^{r}=L^{r}(]-\infty,-t\right] \times \mathbb{R}^{n}\right)$. Strichartz and Hölder estimates yield

$$
\begin{aligned}
\left\|r^{\varepsilon}\right\|_{L_{t, x}^{\gamma}} & \lesssim\left\|\left(\left|\varphi_{0}\right|^{4 / n}+\left|\varepsilon \varphi_{1}\right|^{4 / n}+\left|\varepsilon r^{\varepsilon}\right|^{4 / n}\right) \varepsilon\left(\left|\varphi_{1}\right|+\left|r^{\varepsilon}\right|\right)\right\|_{L_{t, x}^{\gamma^{\prime}}} \\
& \lesssim\left(\left\|\varphi_{0}\right\|_{L_{t, x}^{\gamma}}^{4 / n}+\left\|\varepsilon \varphi_{1}\right\|_{L_{t, x}^{\gamma}}^{4 / n}+\left\|\varepsilon r^{\varepsilon}\right\|_{L_{t, x}^{\gamma}}^{4 / n}\right)\left(\varepsilon\left\|\varphi_{1}\right\|_{L_{t, x}^{\gamma}}+\left\|\varepsilon r^{\varepsilon}\right\|_{L_{t, x}^{\gamma}}\right) \\
& \lesssim\left(\|\phi\|_{L^{2}}^{4 / n}+\varepsilon^{4 / n}\|\phi\|_{L^{2}}^{4 / n+16 / n^{2}}+\left\|\varepsilon r^{\varepsilon}\right\|_{L_{t, x}^{\gamma}}^{4 / n}\right)\left(\varepsilon\|\phi\|_{L^{2}}^{1+4 / n}+\left\|\varepsilon r^{\varepsilon}\right\|_{L_{t, x}^{\gamma}}\right) . \\
& \lesssim \varepsilon+\varepsilon\left\|r^{\varepsilon}\right\|_{L_{t, x}^{\gamma}}+\left\|\varepsilon r^{\varepsilon}\right\|_{L_{t, x}^{\gamma}}^{1+4 / n} .
\end{aligned}
$$

A bootstrap argument shows that for $0<\varepsilon \ll 1, r^{\varepsilon} \in L^{\gamma}\left(\mathbb{R} \times \mathbb{R}^{n}\right)$, and

$$
\left\|r^{\varepsilon}\right\|_{L^{\gamma}\left(\mathbb{R} \times \mathbb{R}^{n}\right)} \lesssim \varepsilon
$$

Using Strichartz estimates again, we infer:

$$
\left\|r^{\varepsilon}\right\|_{L^{\infty}\left(\mathbb{R} ; L^{2}\left(\mathbb{R}^{n}\right)\right)} \lesssim \varepsilon .
$$

Considering $u^{\varepsilon}$ at time $t=0$ yields the first part of the proposition. The second part can be proven in the same way, but can also be inferred from the first part via Neumann series, since $W_{ \pm}$are small perturbations of the identity near the origin.

Now Corollary 1.5 follows from Corollary 1.2 and Proposition 4.1. where we identify the terms of order $\varepsilon^{1+4 / n}$.

Remark 4.2. Considering the asymptotic expansion of the wave operators and their inverse to higher order would yield other formulae, similar to Corollary 1.5 . We have not written them, for they are more intricate (they involve several integrations in time), and we do not know if they can be of some interest.

\section{Appendix A. Alternate proof of Theorem 1.1}

For $x \in \mathbb{R}^{n}$ and $a_{0} \in \Sigma$, consider the semi-classical limit $\varepsilon \rightarrow 0$ for:

$$
i \varepsilon \partial_{t} u^{\varepsilon}+\frac{\varepsilon^{2}}{2} \Delta u^{\varepsilon}=\varepsilon^{2}\left|u^{\varepsilon}\right|^{4 / n} u^{\varepsilon} \quad ; \quad u^{\varepsilon}(t=0, x)=a_{0}(x) e^{-i \frac{|x|^{2}}{2 \varepsilon}} .
$$

Define:

$$
\psi_{-}=e^{-i n \frac{\pi}{4}} \widehat{a}_{0} .
$$

It is proven in 2] (with a different normalization for the Fourier transform), that:

$$
\left\|u^{\varepsilon}(1, \cdot)-\frac{1}{\varepsilon^{n / 2}} \varphi\left(\frac{\cdot}{\varepsilon}\right)\right\|_{\Sigma^{\varepsilon}} \underset{\varepsilon \rightarrow 0}{\longrightarrow} 0,
$$

where $\varphi=W_{-} \psi_{-}, W_{-}$the wave operator associated to 1.1. The norm on $\Sigma^{\varepsilon}$ is:

$$
\|f\|_{\Sigma^{\varepsilon}}=\|f\|_{L^{2}}+\|\varepsilon \nabla f\|_{L^{2}}+\left\|\frac{x}{\varepsilon} f\right\|_{L^{2}} .
$$

It is the usual norm on $\Sigma$, up to an $L^{2}$-unitary rescaling at scale $\varepsilon$. Introduce a "semi-classical" conformal transform (see 44):

$$
u^{\varepsilon}(t, x)=\frac{e^{i \frac{|x|^{2}}{2 \varepsilon(t-1)}}}{(1-t)^{n / 2}} \phi^{\varepsilon}\left(\frac{\varepsilon}{1-t}, \frac{x}{1-t}\right), \quad t<1 .
$$


Then A.1 is equivalent to:

$$
i \partial_{t} \phi^{\varepsilon}+\frac{1}{2} \Delta \phi^{\varepsilon}=\left|\phi^{\varepsilon}\right|^{4 / n} \phi^{\varepsilon} \quad ; \quad \phi_{\mid t=\varepsilon}^{\varepsilon}=a_{0} .
$$

Since 1.1 is globally well-posed in $\Sigma$, we also have:

$$
\left\|U_{0}(-t)\left(\phi^{\varepsilon}(t)-\phi(t)\right)\right\|_{L^{\infty}([0,+\infty[, \Sigma) \underset{\varepsilon \rightarrow 0}{\longrightarrow} 0}
$$

where

$$
i \partial_{t} \phi+\frac{1}{2} \Delta \phi=|\phi|^{4 / n} \phi \quad ; \quad \phi_{\mid t=0}=a_{0} .
$$

On the other hand, asymptotic completeness in $\Sigma$ yields:

$$
\left\|U_{0}(-t) \phi(t)-W_{+}^{-1} a_{0}\right\|_{\Sigma} \underset{t \rightarrow+\infty}{\longrightarrow} 0
$$

Let $\lambda>0$ : we have

$$
u^{\varepsilon}(1-\lambda \varepsilon, x)=\frac{e^{-i \frac{|x|^{2}}{2 \lambda \varepsilon^{2}}}}{(\lambda \varepsilon)^{n / 2}} \phi^{\varepsilon}\left(\frac{1}{\lambda}, \frac{x}{\lambda \varepsilon}\right) .
$$

We infer, by continuity in the rescaled variables:

$$
\begin{aligned}
o_{\lambda \rightarrow 0}(1) & =\limsup _{\varepsilon \rightarrow 0}\left\|u^{\varepsilon}(1)-u^{\varepsilon}(1-\lambda \varepsilon)\right\|_{\Sigma^{\varepsilon}}=\limsup _{\varepsilon \rightarrow 0}\left\|\varphi-\frac{e^{-i \frac{|x|^{2}}{2 \lambda}}}{\lambda^{n / 2}} \phi^{\varepsilon}\left(\frac{1}{\lambda}, \frac{x}{\lambda}\right)\right\|_{\Sigma} \\
& =\left\|\varphi-\frac{e^{-i \frac{|x|^{2}}{2 \lambda}}}{\lambda^{n / 2}} \phi\left(\frac{1}{\lambda}, \frac{x}{\lambda}\right)\right\|_{\Sigma} .
\end{aligned}
$$

But we have:

$$
\left\|U_{0}(t) f-e^{i \frac{|x|^{2}}{2 t}} \frac{1}{(i t)^{n / 2}} \widehat{f}\left(\frac{x}{t}\right)\right\|_{L^{2}} \underset{t \rightarrow+\infty}{\longrightarrow} 0 .
$$

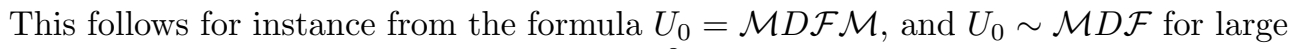
times (see Lemma 2.2. We now consider $L^{2}$-norms:

$$
\begin{aligned}
& \left\|\frac{e^{-i \frac{|x|^{2}}{2 \lambda}}}{\lambda^{n / 2}} \phi\left(\frac{1}{\lambda}, \frac{x}{\lambda}\right)-e^{-i n \frac{\pi}{4}} \mathcal{F}\left(W_{+}^{-1} a_{0}\right)\right\|_{L^{2}}= \\
& =\left\|e^{-i \lambda \frac{|x|^{2}}{2}} \phi\left(\frac{1}{\lambda}, x\right)-\lambda^{n / 2} e^{-i n \frac{\pi}{4}} \mathcal{F}\left(W_{+}^{-1} a_{0}\right)(\lambda x)\right\|_{L^{2}} \\
& =\left\|\phi\left(\frac{1}{\lambda}, x\right)-e^{i \lambda \frac{|x|^{2}}{2}} \lambda^{n / 2} e^{-i n \frac{\pi}{4}} \mathcal{F}\left(W_{+}^{-1} a_{0}\right)(\lambda x)\right\|_{L^{2}} \\
& =\left\|U_{0}\left(-\frac{1}{\lambda}\right) \phi\left(\frac{1}{\lambda}, x\right)-U_{0}\left(-\frac{1}{\lambda}\right) e^{i \lambda \frac{|x|^{2}}{2}} \lambda^{n / 2} e^{-i n \frac{\pi}{4}} \mathcal{F}\left(W_{+}^{-1} a_{0}\right)(\lambda x)\right\|_{L^{2}} \\
& \leqslant o_{\lambda \rightarrow 0}(1)+\left\|W_{+}^{-1} a_{0}-U_{0}\left(-\frac{1}{\lambda}\right) e^{i \lambda \frac{|x|^{2}}{2}} \lambda^{n / 2} e^{-i n \frac{\pi}{4}} \mathcal{F}\left(W_{+}^{-1} a_{0}\right)(\lambda x)\right\|_{L^{2}}=o_{\lambda \rightarrow 0}(1) . \\
& \leqslant o_{\lambda \rightarrow 0}(1)+\left\|U_{0}\left(\frac{1}{\lambda}\right) W_{+}^{-1} a_{0}-e^{i \lambda \frac{|x|^{2}}{2}} \lambda^{n / 2} e^{-i n \frac{\pi}{4}} \mathcal{F}\left(W_{+}^{-1} a_{0}\right)(\lambda x)\right\|_{L^{2}}=
\end{aligned}
$$

We infer:

$$
o_{\lambda \rightarrow 0}(1)=\left\|\varphi-e^{-i n \frac{\pi}{4}} \mathcal{F}\left(W_{+}^{-1} a_{0}\right)\right\|_{L^{2}}
$$


Since the right hand side is independent of $\lambda$, we infer:

$$
\mathcal{F}\left(W_{+}^{-1} a_{0}\right)=e^{i n \frac{\pi}{4}} \varphi=e^{i n \frac{\pi}{4}} W_{-}\left(e^{-i n \frac{\pi}{4}} \widehat{a}_{0}\right)=W_{-} \mathcal{F}\left(a_{0}\right),
$$

which yields Theorem 1.1

\section{Appendix B. Sub-critical case}

In this appendix, we consider more generally the nonlinear Schrödinger equation

$$
i \partial_{t} u+\frac{1}{2} \Delta u=|u|^{2 \sigma} u, \quad(t, x) \in \mathbb{R} \times \mathbb{R}^{n},
$$

in the sub-critical case $\sigma<2 / n$. Following the approach to prove Corollary 1.5, we have:

Proposition B.1. Let $\sigma<2 / n$, with

- $\sigma>1 / n$ if $n \leqslant 2$.

- $\sigma>2 /(n+2)$ if $n \geqslant 2$.

Then the following identities hold for every $\phi \in \Sigma$ :

$$
\begin{aligned}
& \int_{0}^{ \pm \infty} e^{i t \frac{|x|^{2}}{2}} \mathcal{F}\left(\left|U_{0}(t) \phi\right|^{2 \sigma} U_{0}(t) \phi\right) d t=\int_{0}^{ \pm \infty}|t|^{n \sigma-2} U_{0}(t)\left(\left|U_{0}(-t) \widehat{\phi}\right|^{2 \sigma} U_{0}(-t) \widehat{\phi}\right) d t \\
& \int_{0}^{ \pm \infty}|t|^{n \sigma-2} e^{i t \frac{|x|^{2}}{2}} \mathcal{F}\left(\left|U_{0}(t) \phi\right|^{2 \sigma} U_{0}(t) \phi\right) d t=\int_{0}^{ \pm \infty} U_{0}(t)\left(\left|U_{0}(-t) \widehat{\phi}\right|^{2 \sigma} U_{0}(-t) \widehat{\phi}\right) d t .
\end{aligned}
$$

Sketch of the proof. Let $u$ solving (B.1). Then $v=\Psi u$ solves

$$
i \partial_{t} v+\frac{1}{2} \Delta v=|t|^{n \sigma-2}|v|^{2 \sigma} v, \quad(t, x) \in \mathbb{R} \backslash\{0\} \times \mathbb{R}^{n} .
$$

It follows from [7] (see also [5]) that wave operators exist, are continuous and invertible, near the origin in $\Sigma$, both for $($ B.1) and $($ B.2). We can then mimic the proof of Theorem 1.1. with the remark that in Theorem 1.1. the operators $W_{ \pm}^{-1}$ on the left-hand side are associated to $u$, while the operators $W_{\mp}$ on the right-hand side are associated to $v$.

Adapting Proposition 4.1 to the cases of (B.1) and (B.2) proceeds along the same lines as the estimates in [7. This yields the first identity in the above proposition.

For the second, we simply notice that $\Psi^{2}=R$, so that we can exchange the roles of $u$ and $v$.

\section{References}

[1] P. Blue and J. Colliander, Global well-posedness in Sobolev space implies global existence for weighted $L^{2}$ initial data for $L^{2}$-critical NLS, Commun. Pure Appl. Anal. 5 (2006), no. 4, 691708.

[2] R. Carles, Geometric optics with caustic crossing for some nonlinear Schrödinger equations, Indiana Univ. Math. J. 49 (2000), no. 2, 475-551.

[3] —-, Remarques sur les mesures de Wigner, C. R. Acad. Sci. Paris, t. 332, Série I 332 (2001), no. $11,981-984$.

[4] - Cascade of phase shifts for nonlinear Schrödinger equations, J. Hyperbolic Differ. Equ. 4 (2007), no. 2, 207-231.

[5] T. Cazenave, Semilinear Schrödinger equations, Vol. 10 of Courant Lecture Notes in Mathematics, New York University Courant Institute of Mathematical Sciences, New York (2003). 
[6] T. Cazenave and F. Weissler, Some remarks on the nonlinear Schrödinger equation in the critical case, in Lect. Notes in Math., Vol. 1394, 18-29, Springer-Verlag, Berlin (1989).

[7] - Rapidly Decaying Solutions of the Nonlinear Schrödinger Equation, Comm. Math. Phys. 147 (1992) 75-100.

[8] P. Gérard, Oscillations and concentration effects in semilinear dispersive wave equations, J. Funct. Anal. 141 (1996), no. 1, 60-98.

[9] J. Ginibre, An introduction to nonlinear Schrödinger equations, in R. Agemi, Y. Giga, and T. Ozawa, editors, Nonlinear waves (Sapporo, 1995), GAKUTO International Series, Math. Sciences and Appl., 85-133, Gakkōtosho, Tokyo (1997).

[10] J. Ginibre, T. Ozawa, and G. Velo, On the existence of the wave operators for a class of nonlinear Schrödinger equations, Ann. IHP (Physique Théorique) 60 (1994) 211-239.

[11] J. Ginibre and G. Velo, On a class of nonlinear Schrödinger equations. II Scattering theory, general case, J. Funct. Anal. 32 (1979) 33-71.

[12] - Scattering theory in the energy space for a class of nonlinear Schrödinger equations, J. Math. Pures Appl. (9) 64 (1985), no. 4, 363-401.

[13] S. Katayama and Y. Tsutsumi, Global existence of solutions for nonlinear Schrödinger equations in one space dimension, Comm. Partial Differential Equations 19 (1994), no. 11-12, 1971-1997.

[14] T. Ozawa, On the nonlinear Schrödinger equations of derivative type, Indiana Univ. Math. J. 45 (1996), no. 1, 137-163.

[15] T. Ozawa and Y. Tsutsumi, Space-time estimates for null gauge forms and nonlinear Schrödinger equations, Differential Integral Equations 11 (1998), no. 2, 201-222.

[16] M. Tanaka, Nonlinear self-modulation problem of the Benjamin-Ono equation, J. Phys. Soc. Japan 51 (1982), no. 8, 2686-2692.

[17] T. Tao, M. Visan, and X. Zhang, Global well-posedness and scattering for the mass-critical nonlinear Schrödinger equation for radial data in high dimensions, Duke Math. J. 140 (2007), no. 1, 165-202.

[18] Y. Tsutsumi, Scattering problem for nonlinear Schrödinger equations, Ann. Inst. H. Poincaré Phys. Théor. 43 (1985), no. 3, 321-347.

[19] - The null gauge condition and the one-dimensional nonlinear Schrödinger equation with cubic nonlinearity, Indiana Univ. Math. J. 43 (1994), no. 1, 241-254.

CNRS \& Université Montpellier 2, Mathématiques, CC 051, Place Eugène Bataillon, 34095 Montpellier Cedex 5, France

E-mail address: Remi.Carles@math.cnrs.fr

Department of Mathematics, Hokkaido University, Sapporo 060-810, Japan

E-mail address: ozawa@math.sci.hokudai.ac.jp 